COHERENT STATES

\title{
Applications in Physics and
}

Mathematical Physics 
This page is intentionally left blank 


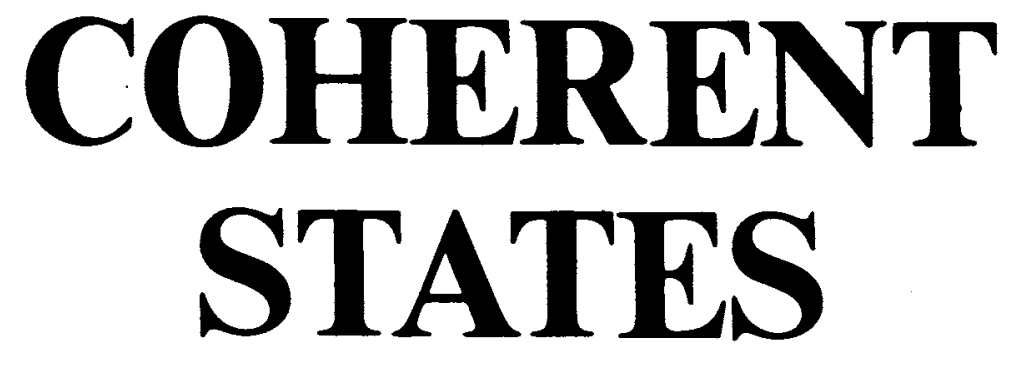

\title{
Applications in Physics and Mathematical Physics
}

\author{
John R. Klauder \\ Bo-Sture Skagerstam
}




\section{Published by}

World Scientific Publishing Co. Pte. Ltd.

P. O. Box 128, Farrer Road, Singapore 9128

The authors and publisher are indebted to the authors and the following publishers of the various journals and books for their assistance and permission to reproduce the selected articles found in this volume:

Academic Press Inc. (Ann. Phys.); American Institute of Physics (Am. J. Phys., J. Math. Phys. and Sov. J. Nucl. Phys.); American Physical Society (Phys. Rev., Phys. Rev. Lett. and Rev. Modern Phys.); Kyoto University (Prog. Theor. Phys. and Prog. Theor. Phys. Suppl.); North-Holland Publishing Co. (Nucl. Phys. and Phys. Lett); Plenum Publishing Co. (Int. J. Theor. Phys. and Symmetries in Science); Physical Society of Japan (J. Phys. Soc. of Japan); Springer-Verlag (Comm. Math. Phys. and Z. Phys. C); The Institute of Physics (J. Phys. A: Gen. Phys. and J. Phys. A: Math. Gen.).

\section{COHERENT STATES - APPLICATIONS IN PHYSICS AND MATHEMATICAL PHYSICS} Copyright (C) 1985 by World Scientific Publishing Co Pte Ltd.

All rights reserved. This book, or parts thereof, may not be reproduced in any form or by any means, electronic or mechanical, including photocopying, recording or any information storage and retrieval system now known or to be invented, without written permission from the Publisher.

ISBN 9971-966-52-2

9971-966-53-0 pbk

Printed in Singapore by Kyodo-Shing Loong Printing Industries Pte Ltd. 


\section{To Our}

\section{Families}


This page is intentionally left blank 


\section{PREFACE}

As a general concept, some notion of coherence applies to nearly every type of phenomenon. This is particularly so for those phenomena involving waves of one kind or another. Indeed, coherence has been recognized as an important feature of optics for more than three centuries, and it is fitting that the concept of coherent states arose in the modern theory of light, quantum optics. Related concepts now permeate many fields of physics and lead to a wide variety of applications.

The purpose of the present volume is to offer a representative collection of articles dealing with coherent states. The reprinted articles are loosely organized by subject matter in Chapters II-XI, and within each chapter they have been chronologically ordered. Our literature searches have revealed over 1000 articles on this subject, and clearly only a small fraction of these could be included. We apologize to those authors whose work we have overlooked and/or who feel their work should have been included.

While the applications of coherent-state methods are quite diverse, there is nevertheless a common core to the subject. In our first chapter, "A Coherent-State Primer", we have attempted to provide an introduction to the variety of definitions and the associated formalism used for coherent states. We hope the reader will find this to be a useful survey, a guide to the various reprints that follow in the remaining chapters, and even an entré to the vast literature on this subject.

The authors thank a number of their colleagues for discussions on the reprinted material and/or their comments on parts or all of the primer, specifically J. P. Blaizot, I. Daubechies, B. DeFacio, R. Gilmore, H. Kuratsuji, E. Lieb, J. Negele, M. Nieto, and T. Suzuki. We also warmly thank Ms. Terry Yulick without whose patience and expertise our task would not have been possible.

Copenhagen, July 1984 
This page is intentionally left blank 


\section{CONTENTS}

Preface

I. A Coherent-State Primer

I.1 Origin and Scope 3

I.2 Coherent States - The Bare Essentials 4

$\begin{array}{ll}\text { I.3 Canonical Coherent States } & 10\end{array}$

I.4 Spin Coherent States 25

I.5 Some Other Coherent States 38

I.6 Coherent-State Path Integrals 60

I.7 Coherent States for Fields 74

\section{REPRINTED PAPERS}

II. General Developments

1. The Action Option and a Feynman Quantization of Spinor Fields in Terms of Ordinary c-Numbers

by J. R. Klauder, Ann. Phys. (N. Y.) 11 (1960) 123

2. Continuous-Representation Theory. I. Postulates of Continuous-Representation Theory by J. R. Klauder, J. Math. Phys. 4 ( 1963 ) 1058

3. Continuous-Representation Theory. II. Generalized Relation Between Quantum and Classical Dynamics by J. R. Klauder, J. Math. Phys. 4 (1963) 1058

4. Photon Correlations

by R. J. Glauber, Phys. Rev. Lett. 10 (1963) 84

5. The Quantum Theory of Optical Coherence by R. J. Glauber, Phys. Rev. 130 ( 1963 ) 2529 
6. Equivalence of Semiclassical and Quantum Mechanical Descriptions of Statistical Light Beams

by E. C. G. Sudarshan, Phys. Rev. Lett. 10 (1963) $277 \quad 199$

7. Coherent and Incoherent States of the Radiation Field by R. J. Glauber, Phys. Rev. 131 (1963) 2766

8. Weak Correspondence Principle

by J. R. Klauder, J. Math. Phys. 8 (1967) 2392

9. Exponential Hilbert Space: Fock Space Revisited by J. R. Klauder, J. Math. Phys. 11 (1970) 609

10. New "Coherent" States Associated with Non-Compact Groups

by A. O. Barut and L. Girardello, Commun. Math. Phys. 21 (1971) 41

11. Some Properties of Coherent Spin States

by J. M. Radcliffe, J. Phys. A: Gen. Phys. 4 (1971) 313

12. Coherent States for Arbitrary Lie Group by A. M. Perelomov, Commun. Math. Phys. 26 (1972) 222

13. A Note on Coherent State Representations of Lie Groups

by E. Onofri, J. Math. Phys. 16 (1975) 1087

14. On Intelligent Spin States

by C. Aragone, E. Chalbaud and S. Salamó, J. Math.

Phys. 17 (1976) 1963

15. Charged Bosons and the Coherent State by D. Bhaumik, K. Bhaumik and B. Dutta-Roy, J. Phys. A: Math. Gen. 9 (1976) 1507

16. Coherent States Associated with the Continuous Spectrum of Noncompact Groups by M. Hongoh, J. Math. Phys. 18 (1977) 2081 
17. Quasi-Coherent States for Unitary Groups by B. -S. Skagerstam, J. Phys. A: Math. Gen. 18 (1985) 1 .

III. Coherent States and Quantum Mechanics

1. Coherent States and the Number-Phase Uncertainty Relation

by P. Carruthers and M. M. Nieto, Phys. Rev. Lett. 14 (1965) 387

2. Coherent States and the Forced Harmonic Oscillator by P. Carruthers and M. M. Nieto, Am. J. Phys. 7 (1965) 537

3. Classical Behavior of Systems of Quantum Oscillators by R. J. Glauber, Phys. Lett. 21 (1966) 650

4. The Classical Limit for Quantum Mechanical Correlation Functions by K. Hepp, Commun. Math. Phys. 35 (1974) 265

5. Quantum Nondemolition Measurement and Coherent States

by W. G. Unruh, Phy. Rev. D17 (1978) 1180

6. Coherent States and Quantum Nonperturbing Measurements

by V. Moncrief, Ann. Phys. (N. Y.) 114 (1978) $201 \quad 362$

7. Coherent States for General Potentials

by M. M. Nieto and L. M. Simmons, Jr., Phys. Rev. Lett. 41 (1978) 207

8. Quantum Limits on Resonant-Mass GravitationalRadiation Detectors

by J. N. Hollenhorst, Phys. Rev. D19 (1979) 1669380 
9. Charged Schrödinger Particle in a c-Number Radiation Field

by T. Toyoda and K. Wildermuth, Phys. Rev. D22 (1980) 2391

10. Large $\mathrm{N}$ Limits as Classical Mechanics

by L. G. Yaffe, Rev. Mod. Phys. 54 (1982) 407

11. Coherent States With assical Motion; From an Analytical Method Complementary to Group Theory by M. M. Nieto in Group Theoretical Methods in Physics, Proceedings, Vol. II, of International Seminar at Zvenigorod, 1982, ed. by M. A. Markov (Nauka, Moscow, 1983 ), p. 174.

IV. Path Integrals and Coherent States

1. Coherent States of Fermi Operators and the Path Integral

by Y. Ohnuki and T. Kashiwa, Prog. Theor. Phys. 60 (1978) 548

2. Path Integrals and Stationary-Phase Approximations by J. R. Klauder, Phys. Rev. D19 (1979) 2349

3. Path Integrals for the Nuclear Many-Body Problem by J. P. Blaizot and H. Orland, Phys. Rev. C24 (1981) 1740

4. Path-Integral Approach to Problems in Quantum Optics

by M. Hillery and M. S. Zubairy, Phys. Rev. A26 (1982) 451

5. Path Integral Approach to Many-Body Systems and Classical Quantization of Time-Dependent Mean Field

by H. Kuratsuji and T. Suzuki, Prog. Theor. Phys. Suppl. 74 \& 75 (1983) 209 
6. Quantum Mechanical Path Integrals with Wiener Measures for all Polynomial Hamiltonians by J. R. Klauder and I. Daubechies, Phys. Rev. Lett. 52 (1984) 1161

V. Pseudospin Techniques

1. Semi-Classical Spectrum of the Continuous Heisenberg Spin Chain

by A. Jevicki and N. Papanicolaou, Ann. Phys. (N. Y.) 120 (1979) 107

2. Bohr-Sommerfeld Quantization of Pseudospin Hamiltonians

by R. Shankar, Phys. Rev. Lett. 45 (1980) 1088

3. Holstein-Primakoff Theory for Many-Body Systems by L. R. Mead and N. Papanicolaou, Phys. Rev. B28 (1983) 1633

VI. Applications in Condensed Matter Physics

1. Coherent States and Irreversible Processes in Anharmonic Crystals

by P. Carruthers and K. S. Dy, Phys. Rev. 147 (1966) 214

2. Theory of Superfluidity

by F. W. Cummings and R. J. Johnston, Phys. Rev. 151 (1966) 105

3. Coherent States in the Theory of Superfluidity by J. S. Langer, Phys. Rev. 167 (1968) 183

4. Landau Diamagnetism from the Coherent States of an Electron in a Uniform Magnetic Field by A. Feldman and A. H. Kahn, Phys. Rev. B1 (1970) 4584 
5. Spin Coherent State Representation in Non-Equilibrium Statistical Mechanics

by Y. Takahashi and F. Shibata, J. Phys. Soc. Japan 38 (1975) 656

6. Coherent-State Langevin Equations for Canonical Quantum Systems with Applications to the Quantized Hall Effect

by J. R. Klauder, Phys. Rev. A29 (1984) 2036

VII. Applications in Thermodynamics

1. The Classical Limit of Quantum Spin Systems

by E. H. Lieb, Commun. Math. Phys. 31 (1973) 327615

2. Coherent States and Partition Function

by M. Rasetti, Int. J. Theor. Phys. 14 (1975) 1

3. Applications of Coherent States in Thermodynamics and Dynamics

by R. Gilmore, in Symmetries in Science, eds. B. Gruber and K. S. Millman (Plenum Publ. Corp., New York, 1980), p. 105

4. On the Large $\mathrm{N}_{\mathrm{c}}$ Limit of the $\mathrm{SU}\left(\mathrm{N}_{\mathrm{c}}\right)$ Colour QuarkGluon Partition Function

by B. -S. Skagerstam, Z. Phys. C24 (1984) 97

VIII. Aplications in Atomic Physics

1. Atomic Coherent States in Quantum Optics

by F. T. Arecchi, E. Courtens, R. Gilmore and $H$. Thomas, Phys. Rev. A6 (1972) 2211

2. Phase Transition in the Dicke Model of Superradiance by Y. K. Wang and F. T. Hioe, Phys. Rev. A7 (1973) 831 
3. Equilibrium Statistical Mechanics of Matter Interacting with the Quantized Radiation Field

by K. Hepp and E. H. Lieb, Phys. Rev. A8 (1973) 2517

4. Multitime-Correlation Functions and the Atomic Coherent-State Representation by L. M. Narducci, C. M. Bowden, V. Bluemel, G. P. Garrazana and R. A. Tuft, Phys. Rev. A11 (1975) 973

IX. Applications in Nuclear Physics

1. Coherent States of the Quantum-Mechanical Top by D. Janssen, Sov. J. Nucl. Phys. 25 (1977) 479

2. Studies of the Ground-State Properties of the LipkinMeshkov-Glick Model via the Atomic Coherent States by R. Gilmore and D. H. Feng, Phys. Lett. 76B (1978) 26

3. Phase Transitions in Nuclear Matter Described by Pseudospin Hamiltonians

by R. Gilmore and D. H. Feng, Nucl. Phys. A301 (1978) 189

4. Condensates and Bose-Einstein Correlations

by G. N. Fowler, N. Stelte and R. M. Weiner, Nucl. Phys. A319 (1979) 349

X. Applications in Elementary Particle Physics

1. Coherent Production of Pions

by D. Horn and R. Silver, Ann. Phys. (N. Y.) 66 (1971) 509

2. Coherent States and Particle Production

by J. C. Botke, D. J. Scalapino and R. L. Sugar, Phys. Rev. D9 (1974) 813 
3. Coherent Quark-Gluon Jets

by G. Curci, M. Greco and Y. Srivastava, Phys. Rev. Lett. 43 (1979) 834

4. Coherent-State Representation of a Non-Abelian Charged Quantum Field

by K. - E. Eriksson, N. Mukunda and B. -S. Skagerstam, Phys. Rev. D24 (1981) 2615

5. Correlations and Fluctuations in Hadronic Multiplicity Distributions: The Meaning of the KNO Scaling by P. Carruthers and C. C. Shih, Phys. Lett. 127B (1983) 242

6. Hadronic Multiplicity Distribution: Example of a Universal Stochastic Process

by P. Carruthers, in Proceedings of the XIVth International Symposium on Multiparticle Dynamics, eds. P. Yager and J. F. Gunion (World Scientific Publishing Co., 1984), p. 825

XI. Applications in Quantum Field Theory

1. Infrared Divergences in Quantum Electrodynamics by V. Chung, Phys. Rev. 140 (1965) B1.110

2. Coherent States and Indefinite Metric - Applications to the Free Electromagnetic and Gravitational Fields by J. Gomatam, Phys. Rev. D3 (1971) 1292

3. Extended Particles and Solitons by K. Cahill, Phys. Lett. 53B (1974) 174

4. Classical Source Emitting Self-Interacting Bosons by K. -E. Eriksson and B. -S. Skagerstam, Phys. Rev. D18 ( 1978) 3858

5. Path-Integral Representation for the S Matrix by C. L. Hammer, J. E. Shrauner and B. De Facio, Phys. Rev. D18 (1978) 373 
6. Coherent State Approach to the Infra-red Behaviour of Non-Abelian Gauge Theories

by M. Greco, F. Palumbo, G. Pancheri-Srivastava and Y. Srivastava, Phys. Lett. 77B (1978) 282

7. Creation and Annihilation Operators for NielsenOlesen Vortices in the Coherent State Approximation by D. E. L. Pottinger, Phys. Lett. 78B (1978) 476902

8. Vacuum Instability and the Critical Value of the Coupling Parameter in Scalar QED by G. -J. Ni and Y. -P. Wang, Phys. Rev. D27 (1983) 969 